Original Article

\title{
INTRANASAL MICROEMULGEL AS SURROGATE CARRIER TO ENHANCE LOW ORAL BIOAVAILABILITY OF SULPIRIDE
}

\author{
ABDALLAH MOHAMED AYOUB', MAHMOUD MOKHTAR IBRAHIM ${ }^{1,2}$, MARWA HELMY ABDALLAH ${ }^{1,3}$, \\ MAHMOUD A. MAHDY1 \\ 1Dept. of Pharmaceutics, Faculty of Pharmacy, Zagazig University, Zagazig, Egypt, ${ }^{2}$ Dept. of Pharmaceutics, Faculty of Pharmacy, Delta \\ University, Mansoura, Egypt, ${ }^{3}$ Department of Pharmaceutics, College of Pharmacy, Hail University, Hail, Kingdom of Saudi Arabia \\ Email: mahmoktar@yahoo.com
}

Received: 30 Jun 2016 Revised and Accepted: 12 Aug 2016

\begin{abstract}
Objective: The purpose of this study is to evaluate microemulsion based gel (MBG) of sulpiride "a poorly water soluble antipsychotic with low oral bioavailability."

Methods: Gelling polymers such as sodium carboxymethylcellulose (CMC-Na), hydroxyl propyl methyl cellulose (HPMC K4m), carbopol 940 and Na alginate were evaluated for their potential to gel sulpiride microemulsions (MEs) without affecting the MEs structure. Also, sulpiride solution (SS) and conventional gel (without ME) were prepared and compared with MBG. Gel formulations were checked for their viscosity, pH, spreadability (S), mucoadhesive force (MF), and nasal ciliotoxicity studies. The in vitro release of sulpiride across a cellophane membrane and its permeation through the nasal mucosa in phosphate buffered saline pH 6.8 (PBS) were also performed. In addition, a pharmacodynamic study of optimized formulae compared to SS and microemulsion (ME) was evaluated in rats.
\end{abstract}

Results: CMC-Na and HPMC K4m were not able to gel sulpiride loaded MEs while Na alginate gave an unclear gel with a sticky texture. Results revealed that the viscosity, mucoadhesion force, and spreadability of the MBG increased with increasing carbopol 940 concentrations. The flux was arranged as the following, MBG>conventional gel>sulpiride solution (SS). According to histopathological study, safe and non-irritant MBGs suitable for nasal administration were successfully prepared. Finally, the pharmacodynamic study indicated that intranasal sulpiride MBG had a significant effect $(* \mathrm{P}<0.001)$ than SS and ME administered either intravenous or intranasal.

Conclusion: MBG provides significant enhancement in nasal bioavailability not only by absorption enhancing effect of ME but also, by increasing nasal residence time

Keywords: Microemulsion, Sulpiride, Mucoadhesive, Gels, Intranasal delivery, Microemulgel, carbopol 940

(C) 2016 The Authors. Published by Innovare Academic Sciences Pvt Ltd. This is an open access article under the CC BY license (http://creativecommons.org/licenses/by/4. 0/) DOI: http://dx.doi.org/10.22159/ijpps.2016v8i10.13776

\section{INTRODUCTION}

Mucociliary clearance (MCC) decreases the contact of the intranasal formulations with the mucosal membrane, hence, active drugs get fast cleared from the nasal cavity and less drug amount could be absorbed [1]. Nasal gels are highly viscous solutions or suspensions that reduce the post-nasal drip, reduce the taste impact due to reduced swallowing, reduce the anterior leakage of the formulation, reduce the irritation due to soothing/emollient excipients and target delivery to nasal mucosa resulting in a better drug absorption [2]. When gel formula and microemulsion (ME) are combined together, the produced dosage form is referred as microemulsion based gel (MBG) or micro emulged. Micro emulgel enhances the adhesion of the optimized ME formulation and prolongs its retention time after application, consequently, it is more suitable for topical application when compared with ME liquid formula [3].

Sulpiride "Dogmatil ${ }^{\circledR}$ a model for benzamide atypical antipsychotic drugs is a selective antagonist to dopamine (D2) receptors. It is used in the treatment of major depressive disorders and in psychosis associated with schizophrenia. Moreover, it is used in the treatment of anxiety, gastric and duodenal ulcers and mild depression disorders [4]. Sulpiride is classified under class IV in Biopharmaceutical classification system (BCS). This means that sulpiride has poor aqueous solubility $(0.5 \mathrm{mg} / \mathrm{ml})$ and limited permeability. The drug may be a substrate for P-gp back-efflux. It showed a variable bioavailability after oral administration due to its poor water solubility. The oral bioavailability of sulpiride is reported as low as in human and rats " $27 \%$ and $15-20 \%$, respectively" where it was relatively higher in dogs (about 44-85\%). The poor intestinal bioavailability of sulpiride was due to poor absorption rates which could be increased by the concomitant use of P-glycoprotein (P-gp) substrates such as cyclosporine A [4].
Since the target site of the sulpiride is the brain, thereby a strategy is desirable to improve the bioavailability by overcoming low solubility/dissolution rate, low permeability, and P-gp efflux. This decreases the dose and frequency of dosing and possibly maximizes the therapeutic index. Hence, preventing the availability of drug at non-targeting sites and reducing the side effects.

The aim of the present investigation is to formulate and evaluate intranasal sulpiride micro-emulgelsin order to increase the nose to brain drug delivery that could reduce the drug dosage and side effects and maintain patient compliance with the final goal of improving the therapeutic outcome.

\section{MATERIALS AND METHODS}

\section{Materials}

Sulpiride was kindly supplied by Delta pharm. Co., Egypt. Cremophor RH-40 (Polyoxyl 40 hydrogenated castor oil) and carbo pol 940 were kindly supplied by sigma pharm. Co., Egypt. Labrasol (caprylocaproylmacrogol-8-glyceride), labrafil isostearique (triisostearin PEG-6esters), peceol (glyceryl monooleate (GMO)) and transcutol HP (highly purified diethylene glycol monoethyl ether) were kindly supplied by Gattefossé, Saint-Priest, France. Absolute ethanol, polyethylene glycol 400 (PEG 400) and tween 20 (T20) were purchased from El-Gomheria pharm. Co., Egypt. Sodium carboxymethylcellulose, triethanolamine, sodium alginate, xanthan gum and carbo pol 971 were donated as a gift from EIPICO, $10^{\text {th }}$ of Ramadan City, Egypt. All other chemicals were of analytical grade.

Preparation of mucoadhesive gels

Different thickening polymers, namely, carbopol 971, sodium carboxymethylcellulose "CMC-Na", carbopol 940, HPMC K4m, 
sodium alginate, and xanthan gum were evaluated for their potential to gel sulpiride MEs. In Brief, MEs were prepared with minimum water content. Each thickening polymer was gelled with distilled water separately using a magnetic stirrer (Heidolph PZP-200, Germany) at $1000 \mathrm{rpm}$ for $30 \mathrm{~min}$. The aqueous gel dispersion was added in a dropwise manner to sulpiride ME under magnetic stirring to obtain MBGs. The prepared MBGs were kept in closed containers at room temperature for $24 \mathrm{~h}$ then investigated visually for phase separation or turbidity. A better gelling polymer was selected on the basis of compatibility with ME components, ease of gel spreadability and no signs of phase separations $[5,6]$.

\section{Preparation of mucoadhesive plain gel (Conventional gel)}

Carbopol 940 was dispersed in a sufficient quantity of distilled water using a magnetic stirrer for $30 \mathrm{~min}$ to get viscous homogeneous dispersions. The prepared dispersions were kept in the dark and at 4 $\mathrm{C}$ for $24 \mathrm{~h}$ for complete swelling of carbopol. Sulpiride representing $0.5 \%(\mathrm{w} / \mathrm{w})$ was dissolved in ethanol/PEG 400 (2:3) mixed solvent then added to the aqueous polymer dispersions. Triethanolamine was added in dropwise manner to $\mathrm{pH}$ values between 6 and 6.5 using a pH electrode (Jenway 3510, UK) dipping into the formulation and finally, a semisolid gel-like consistency was obtained [7].

\section{Preparation of mucoadhesive microemulsion based gel (MBG)}

Here, the influence of order of addition of carbopol 940 on the formation of MBG was also investigated. In one case, an aqueous dispersion of carbopol 940 , equivalent to $0.5,1,1.5$ and $2 \% \mathrm{w} / \mathrm{w}$ of the formulation in a sufficient quantity of distilled water (gelling phase) were prepared. The dispersions were kept at $4{ }^{\circ} \mathrm{C}$ for $24 \mathrm{~h}$ for complete swelling of the polymer. The aqueous dispersions of carbopol 940 were slowly mixed with sulpiride ME under stirring at $1000 \mathrm{rpm}$ and were allowed to homogenize for $10 \mathrm{~min}$; then the mixtures were titrated with triethanolamine to $\mathrm{pH}$ 6-6.5 to obtain MBGs $[8,9]$.

In the second case, carbopol 940 was added directly to the preformed sulpiride ME by stirring to prepare MBG without being swollen gel matrix [6, 10]. Briefly, a weighed amount of carbopol 940 was directly added to ME system, stirred for $30 \mathrm{~min}$ to disperse the polymer in the ME dispersion and left overnight to obtain gels. The required quantity of triethanolamine was added for neutralizing the carboxylic acid groups in carbopol and the $\mathrm{pH}$ was adjusted between 6 and $6.5[6,11]$. The composition of mucoadhesive conventional gel and microemulgel formulae was shown in table 1.

\section{Preparation of sulpiride solution (SS)}

The SS was prepared by dissolving sulpiride (50 $\mathrm{mg}$ ) in a mixture of ethanol (95\%) and PEG 400 in $1: 2 \mathrm{w} / \mathrm{w}$ ratios. Finally, the volume was adjusted to $5 \mathrm{ml}$ with phosphate buffer saline of $\mathrm{pH}$ 6.8 (PBS) with stirring for $10 \mathrm{~min}$ resulting in a clear solution of 10 $\mathrm{mg} / \mathrm{ml}[12]$.

\section{Characterization of MBG}

\section{Physical appearance}

The prepared formulations were inspected visually for their color, homogeneity, appearance of any aggregates, consistency and phase separation $[13,14]$.

\section{PH}

Each sample (1g) was subjected to 10 times dilution with purified water before analysis. The electrode of the $\mathrm{pH}$ meter (Jenway 3510, UK) was immersed in the prepared solution for $\mathrm{pH}$ determination $[13,15]$.

Table 1: Compositions of MEs, mucoadhesive conventional gel and MMBG*Components in the table is presented in gram (g)

\begin{tabular}{|c|c|c|c|c|c|c|c|c|c|}
\hline $\mathbf{F}$ & GMO & T20 & Ethanol & Labrafil & Labrasol & Cremophor & Transcutol & Carbopol-940 & Water \\
\hline F1 & 1.75 & 10.5 & 3.5 & - & - & - & - & - & to $25 \mathrm{~g}$ \\
\hline $\mathrm{F} 2$ & - & - & - & 1.75 & 7 & 7 & - & - & to $25 \mathrm{~g}$ \\
\hline F3 & - & - & - & 1.75 & 3.5 & 3.5 & 7 & - & to $25 \mathrm{~g}$ \\
\hline $\mathrm{F} 4$ & - & - & - & 1.75 & - & 7 & 7 & - & to $25 \mathrm{~g}$ \\
\hline F5 & 1.75 & 10.5 & 3.5 & - & - & - & - & 0.125 & to $25 \mathrm{~g}$ \\
\hline F6 & 1.75 & 10.5 & 3.5 & - & - & - & - & 0.25 & to $25 \mathrm{~g}$ \\
\hline F7 & 1.75 & 10.5 & 3.5 & - & - & - & - & 0.375 & to $25 \mathrm{~g}$ \\
\hline F8 & 1.75 & 10.5 & 3.5 & - & - & - & - & 0.5 & to $25 \mathrm{~g}$ \\
\hline F9 & - & - & - & 1.75 & 7 & 7 & - & 0.125 & to $25 \mathrm{~g}$ \\
\hline F10 & - & - & - & 1.75 & 7 & 7 & - & 0.25 & to $25 \mathrm{~g}$ \\
\hline F11 & - & - & - & 1.75 & 7 & 7 & - & 0.375 & to $25 \mathrm{~g}$ \\
\hline F12 & - & - & - & 1.75 & 7 & 7 & - & 0.5 & to $25 \mathrm{~g}$ \\
\hline F13 & - & - & - & 1.75 & 3.5 & 3.5 & 7 & 0.125 & to $25 \mathrm{~g}$ \\
\hline F14 & - & - & - & 1.75 & 3.5 & 3.5 & 7 & 0.25 & to $25 \mathrm{~g}$ \\
\hline F15 & - & - & - & 1.75 & 3.5 & 3.5 & 7 & 0.375 & to $25 \mathrm{~g}$ \\
\hline F16 & - & - & - & 1.75 & 3.5 & 3.5 & 7 & 0.5 & to $25 \mathrm{~g}$ \\
\hline F17 & - & - & - & 1.75 & - & 7 & 7 & 0.125 & to $25 \mathrm{~g}$ \\
\hline F18 & - & - & - & 1.75 & - & 7 & 7 & 0.25 & to $25 \mathrm{~g}$ \\
\hline F19 & - & - & - & 1.75 & - & 7 & 7 & 0.375 & to $25 \mathrm{~g}$ \\
\hline $\mathrm{F} 20$ & - & - & - & 1.75 & - & 7 & 7 & 0.5 & to $25 \mathrm{~g}$ \\
\hline F21 & - & - & - & - & - & - & - & 0.125 & to $25 \mathrm{~g}$ \\
\hline F22 & - & - & - & - & - & - & - & 0.25 & to $25 \mathrm{~g}$ \\
\hline F23 & - & - & - & - & - & - & - & 0.375 & to $25 \mathrm{~g}$ \\
\hline F24 & - & - & - & - & - & - & - & 0.5 & to $25 \mathrm{~g}$ \\
\hline
\end{tabular}

GMO: peceol (glyceryl-monooleate), T20: tween 20

\section{Drug content measurement}

An accurately weighed amount of MBG "0.5 g" was dissolved in 10 $\mathrm{ml}$ methanol and the mixture was mixed thoroughly using a vortex mixer (16700 mixers, thermoline corporations, Dubuque lowa, USA) for 5 min to ensure complete solubilization of ME components and complete extraction of sulpiride. Subsequently, the solution was filtered using membrane filter $(0.45 \mu \mathrm{m})$ and the drug content in the filtrate was estimated spectrophotometrically (Geneysis-UV-1201, USA), at $290 \mathrm{~nm}$. The plain MBG formulations with the same composition, without the drug, was taken as a blank after appropriate dilution with methanol to cancel out interferences [10, 16].

\section{Spreadability study}

The gel spreadability was estimated as follows: About "1 g"gel was placed within a $1 \mathrm{~cm}$ diameter pre-marked circle on a glass plate over which a second glass plate was placed. A weight of $500 \mathrm{~g}$ was allowed to rest on the upper glass plate for 5 min until no more 
spreading was expected. The circle diameter of spread gel was measured and the mean was taken by repeating the experiment three times. The increase in the diameter $(\mathrm{cm} / \mathrm{g})$ due to spreading of the gels was used as comparative values for spreadability $[6,10]$.

\section{Rheological study}

The viscosity of MBGs and conventional gel formulations, in centipoise ( $\mathrm{Cp}$ ), were determined at room temperature using a Brookfield viscometer (Brookfield DV model DV-II+viscometer, Brookfield Engineering laboratories, Inc., Stoughton, U. S. A) Spindle no 6 was used and $\mathrm{rpm}$ set at 1.5. Spindle was lowered perpendicularly into the center of MBG taking care that spindle does not touch the jar bottom [15].

\section{Determination of mucoadhesive force (MF)}

The modified balance technique using two glass vials and sheep nasal mucosa was used [17]. The mucoadhesive potential of micro emulges and the conventional gel was determined by measuring the force required to detach the formula from nasal mucosal tissue in between two vials using a modified balance developed in our lab as in fig. 1.

In brief, nasal tissues were carefully removed from the nasal cavity of sheep obtained from the local slaughterhouse. Tissues were immediately used after separation. The fresh sheep nasal mucosa was cut into pieces $\left(2.5 \mathrm{~cm}^{2}\right)$ and washed with PBS. Two pieces of the nasal mucosa (E) were secured, keeping the mucosal side out, to the two glass vials $(\mathrm{C})$ separately using cyanoacrylate adhesive and a rubber band. One vial was fixed on the height adjustable pan (F) and the other vial was tied with the balance (A) on the right-hand side. About $0.5 \mathrm{~g}$ gel was applied on the nasal tissue between these two vials. The height of vial adjusted so that the gel could adhere to the mucosal surface of both vials then applied pressure to remove entrapped air. A constant weight was placed on the upper vial and applied for $2 \mathrm{~min}$, after which it was removed, and the upper vial was connected to the balance. Water was added slowly at a constant rate to a plastic jar placed on the left-hand pan (a rate of 13-15 drops per min) until the both vials were separated [17]. The mucoadhesive force, expressed as the detachment stress in dynes $/ \mathrm{cm}^{2}$, was determined using the following equation:

$$
\text { Detachment stress }\left(\text { dynes } / \mathrm{cm}^{2}\right)=\mathrm{mg} \text { A [17] }
$$

Where $\mathrm{m}$ is the minimum weight of water (g) that detached the two vials; $g$ is the acceleration due to gravity taken as $980 \mathrm{~cm} / \mathrm{sec}^{2}$; $\mathrm{A}\left(\mathrm{cm}^{2}\right)$ is the area of the tissue exposed and is equal to $\pi r^{2}(r$, the radius of the exposed tissue).

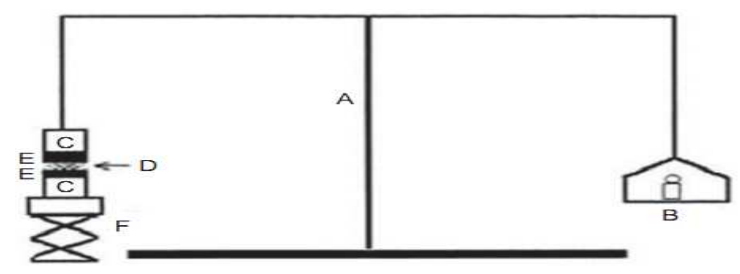

Fig. 1: Modified chemical balance for measuring mucoadhesive force (A) modified balance; (B) plastic jar; (C) glass vial; (D) gel formulation; (E) tissue; (F) height-adjustable pan [17]

\section{Nasal ciliotoxicity study}

Nasal ciliotoxicity studies were carried out using excised sheep nasal mucosa to exclude any histological changes in nasal mucosa caused by formulations. Freshly excised sheep nasal mucosa, except for the septum, was collected from the slaughterhouse in PBS (pH, 6.8). Three sheep nasal mucosa pieces (P1, P2, and P3) with uniform thickness were selected. P1 was treated with $0.5 \mathrm{ml}$ of saline (as a negative control), P2 with $0.5 \mathrm{ml}$ of isopropyl alcohol (a strong mucociliary toxin as a positive control), and P3 was treated with F14 (optimized formula). After $1 \mathrm{~h}$, the mucosa was rinsed with PBS (pH, 6.8) and fixed in $10 \%$ neutral buffered formalin overnight, cut vertically, dehydrated with a graded series of ethanol. These pieces of mucosa were embedded in paraffin blocks, and fine sections $(3 \mu \mathrm{m}$ in thickness) were taken with the microtone and stained with hematoxylin and eosin. The sections were examined and photographed using Nikon Optiphot microscope (Nikon Fx-35A, Nikon Instruments Inc., Japan) (magnification $\times 400$ ) [18].

\section{In vitro release of sulpiride from different MBG}

The in vitro release was determined using the simple dialysis method with some modifications [7]. In this method, $2 \mathrm{~g}$ of the tested formulation, containing $10 \mathrm{mg}$ of sulpiride, was accurately weighed over a plastic cup. The surface of weighing formulation was made flat; the cups were fitted with the dialysis sheet (the cellophane membrane which previously soaked in distilled water for $15 \mathrm{~min}$ ) with a rubber band over the end of a glass tube with an external diameter of $2.9 \mathrm{~cm}$, an internal diameter of $2.6 \mathrm{~cm}$ and a length of 30 $\mathrm{cm}$ (the donor). The diffusion cell was placed at the center of $250 \mathrm{ml}$ beaker containing $100 \mathrm{ml}$ of PBS (pH 6.8) in a water bath shaker (Kotterman Shaker D3165 Hangisen, W-Germany). The stirring rate was $50 \mathrm{rpm}$ and the temperature was maintained at $37 \pm 0.5^{\circ} \mathrm{C}$. At suitable time intervals $(0.5,1,2,3,4,5,6,7,8$ and $24 \mathrm{~h}) ; 2 \mathrm{ml}$ samples were withdrawn from the sink solution and replaced with an equivalent amount of the fresh release medium kept at $37{ }^{\circ} \mathrm{C}$, samples were assayed spectrophotometrically at $293 \mathrm{~nm}$ using the fresh release medium as a blank. Each experiment was done in triplicate and the average cumulative amount was calculated.

\section{Ex-vivo diffusion study}

The ex-vivo diffusion studies of sulpiride loaded ME and drug solution was carried out using freshly isolated sheep nasal mucosa collected from the slaughterhouse. The nasal membrane was kept in PBS (pH, 6.8) for $15 \mathrm{~min}$ to equilibrate [19]. The sheep nasal mucosal membrane (surface area $=2.54 \mathrm{~cm}^{2}$ ) was fixed at one end of the cell immersed in $250 \mathrm{ml}$ beaker containing $100 \mathrm{ml}$ PBS (pH, 6.8) in the presence of $0.02 \%$ sodium azide as a preservative. The diffusion cells were kept in a thermostatic water bath stirred at $100 \mathrm{rpm}$ and the temperature was maintained at $37 \pm 0.5{ }^{\circ} \mathrm{C}$ and complete as mentioned in in vitro release. The cumulative amount of sulpiride permeated across the sheep nasal mucosa " $\mathrm{Q}_{\mathrm{n}}$ " was determined using the following equation [20].

$$
Q_{n}=\frac{C_{n} \times V_{0}+\sum_{i=1}^{n-1} C_{i} \times V_{i}}{S}
$$

Where, $C_{n}$ : the drug concentration after each sampling time, $C_{i}$ : the drug concentration for $\mathrm{i}^{\text {th }}$ sample, $\mathrm{V}_{0}$ and $\mathrm{V}_{\mathrm{i}}$ : the volumes of the receiver solution and sample, respectively,

\section{S: the effective diffusion area.}

\section{Stability studies}

Stability was monitored at refrigerated conditions $\left(2-8{ }^{\circ} \mathrm{C}\right)$, ambient temperature $\left(37^{\circ} \mathrm{C}\right)$ and $50^{\circ} \mathrm{C}[10]$. At the time intervals of $1,2,3$ and 6 mo of storage, the formulations were tested for physical stability (phase separation or flocculation), the appearance, drug content, $\mathrm{pH}$, and viscosity. Also, the formulations were characterized for accelerated centrifugation cycle (13000 rpm for $15 \mathrm{~min}$ ) [21].

\section{Pharmacodynamic study (paw test)}

Wistar Albino male rats weighing between 200 and 250 g were obtained from the animal breeding center, Faculty of Veterinary Medicine, Zagazig University, Egypt and treated according to the Ethical Committee of animal handling in Faculty of pharmacy Zagazig University "ECAHAZU" (Permission number is P2-1-2016). The rats were housed in clean and dry polycarbonate cages and maintained in a well-ventilated room at $25 \pm 1{ }^{\circ} \mathrm{C}$ temperature and $45-55 \% \pm 5 \%$ humidity measured with a digital hygrometer until the day prior to the experiment. The rats were maintained on a standard $12 \mathrm{~h}$ light-dark cycle, and had free access to food and water, except during the experiment. Three rats for each formulation per time point were used in the study. The drug formulation, sulpiride MEs (equivalent to $10 \mathrm{mg} / \mathrm{kg}$ body weight) was injected into the tail vein of rats. Similarly, normal saline (control), sulpiride solution (SS) and sulpiride $\mathrm{ME}$ formulations (equivalent to $10 \mathrm{mg} / \mathrm{kg}$ body weight) 
were administered as $50 \mu \mathrm{l}$ dose "containing $1 \mathrm{mg}$ " of the drug in each nostril using micropipette $(10-100 \mathrm{ml})$ attached with lowdensity polyethylene (LDPE) tubing, having $0.1 \mathrm{~mm}$ internal diameter at the delivery site. Haloperidol (standard) was given in a dose of $0.5 \mathrm{mg} / \mathrm{kg}$ of body weight intraperitoneally (IP) $30 \mathrm{~min}$ before administration of the formulations [22]. The paw test was performed using a Perspex platform measuring $30 \mathrm{~cm} \times 30 \mathrm{~cm}$, with a height of $20 \mathrm{~cm}$. The top of the platform had two holes of $40 \mathrm{~mm}$ diameter for the forelimbs, two holes of $50 \mathrm{~mm}$ diameter for the hind limbs and a slit for the tail. The distance between the right and the left forelimb and hind limb holes was $15 \mathrm{~mm}$, and the distance between forelimb and hind limb holes was $55 \mathrm{~mm}$. The test was performed $30 \mathrm{~min}$ after the intranasal administration of the saline (control group) or the drug formulations by carefully lowering the hind limbs of the rats in the holes, followed by the forelimbs (positioning first the hind and then the forelimbs in the holes). The Forelimb Retraction Time (FRT) was defined as the time it took the rat to withdraw either of the forelimbs. Likewise, the Hind limb Retraction Time (HRT) was defined as the time it took the rat to withdraw either of the hind limbs. The paw test was repeated at 40 and $50 \mathrm{~min}$ after the administration of the formulations [23].

\section{Statistical analysis}

ANOVA "one-way analysis of variance" followed by the LSD as a posthoc test was applied, using SPSS program version 9 software. The differences were considered significant if $\mathrm{P}<0.001$.

\section{RESULTS AND DISCUSSION}

\section{MBG formulation}

MEs of the o/w type, destined to be incorporated into a hydrophilic gel, were dissolved in the aqueous gel phase. The oily phase amount in the ME should be enough to allow the complete drug solubilization if it is lipophilic [24]. After addition of ME to gelling agents for $24 \mathrm{~h}$, carbopol 940 remained clear, however, cellulose derivatives as CMC-Na and HPMC K4m were not able to gel sulpiride loaded MEs. This inefficiency of cellulose polymers could be attributed to their susceptibility to coagulate in the presence of high surfactant concentrations as in the case of ME [10,25]. On the other hand, sodium alginate hydrogel gave turbid, highly viscous with sticky texture gel when combined with MEs. Also, it was noticed that sodium alginate had affected the MEs structure and resulted in oil phase separation [26]. Carbopol 940 MBGs gave clear gels with desired rheological property without disturbing the microstructure or causing phase separations of sulpiride MEs. Carbopol 940 was selected as a gelling agent based on preliminary experiments and previous reports due to its widespread use in pharmaceutical formulations and fast dispersion in water [26,27]. Moreover, it is speculated that carbopol with low concentration can be safe for nasal administration [17].

In this study, the influence of order of addition of carbopol 940 on the formation of MBG was also investigated. In the first case, carbopol 940 was swollen in the aqueous phase for $24 \mathrm{~h}$ and then it was incorporated in the oily phase containing a surfactant, cosurfactant, and drug resulting in a homogenous MBG formulation after $\mathrm{pH}$ was adjusted with triethanolamine. Formulations with no agglomerate were observed due to complete swelling of the polymer [21]. In the second case, carbopol 940 was added directly to the preformed ME without previous swelling in aqueous phase [25]. It took much more time to be swollen in ME than in water (aqueous phase). However, some tiny agglomerates of carbopol 940 were observed as it was not properly swollen. It might attribute to the relatively high viscosity of ME and more time was consumed for the swelling of the polymer [21]. Moreover, this system showed a poor stability, non-uniformity and more air bubbles and lumps, when compared with MBG, swelled in water [11]. In preparing gels, the color of the final formulation was turned ivory white. This was caused by dehydration of some of the ingredients like surfactant and co-surfactant of ME, which results in dissociation of polymers from the hydrated state [21]. Based on these studies, MBG was prepared by mixing the swollen gel matrix (the aqueous phase) with sulpiride ME to form homogenous microemulgel in the subsequent study [28].

\section{Characterization of the prepared gel formulations}

\section{Physical appearance}

To observe the consistency and homogeneity of the gel, a small quantity of gel was pressed between the thumb and index finger, and it was observed that there were no coarse particles in the optimized gel formulation [27]. The conventional hydrogels were aesthetically appealing in appearance and free of entrapped air. They appeared from transparent to translucent and free of lumps. The prepared microemulgel were yellow viscous clear gel preparation with a smooth and homogeneous appearance. There were no signs of phase separation for $24 \mathrm{~h} \mathrm{[13].} \mathrm{The} \mathrm{gel} \mathrm{containing} 0.5 \% \mathrm{w} / \mathrm{w}$ carbopol 940 had a relatively high fluidity, and a weak gel behavior is exhibited by the aqueous carbopol system [29].

Table 2: Physicochemical characterization of conventional gel and MMBG

\begin{tabular}{|c|c|c|c|c|c|}
\hline $\mathbf{F}$ & Drug content $(\%)$ & pH & $S(\mathbf{c m})$ & MF(dyne $\left./ \mathrm{cm}^{2}\right)$ & Viscosity (Cp) \\
\hline F1 & $98.11 \pm 2.4$ & $5.12 \pm 0.18$ & - & - & $28 \pm 6.08$ \\
\hline $\mathrm{F} 2$ & $98.08 \pm 1.55$ & $4.72 \pm 0.11$ & - & - & $257 \pm 12.12$ \\
\hline F3 & $97.15 \pm 1.1$ & $4.25 \pm 0.33$ & - & - & $181 \pm 23.26$ \\
\hline $\mathrm{F} 4$ & $97.76 \pm 2.1$ & $5.74 \pm 0.24$ & - & - & $141 \pm 29.82$ \\
\hline F5 & $97.6 \pm 0.84$ & $7.02 \pm 0.06$ & $10.17 \pm 0.76$ & $471.67 \pm 53.46$ & $37.67 \pm 5.5$ \\
\hline F6 & $95.45 \pm 1.48$ & $6.8 \pm 0.1$ & $8.97 \pm 0.25$ & $994.2 \pm 80.39$ & $66.7 \pm 4.16$ \\
\hline F7 & $98.45 \pm 2.76$ & $6.81 \pm 0.07$ & $8.2 \pm 0.2$ & $1509.33 \pm 140.93$ & $144.33 \pm 10.07$ \\
\hline F8 & $95.75 \pm 3.18$ & $6.77 \pm 0.25$ & $7.63 \pm 0.15$ & $2842 \pm 85.16$ & $1336 \pm 80.82$ \\
\hline F9 & $97.05 \pm 1.9$ & $6.43 \pm 0.35$ & $9 \pm 0.3$ & $948 \pm 87.43$ & $783 \pm 20.07$ \\
\hline F10 & $96.75 \pm 1.77$ & $6.53 \pm 0.67$ & $8.03 \pm 0.15$ & $2598.6 \pm 281.86$ & $4560 \pm 54.4$ \\
\hline F11 & $98.2 \pm 1.27$ & $6.55 \pm 0.42$ & $7.43 \pm 0.12$ & $3392.67 \pm 355.92$ & $25700 \pm 1044$ \\
\hline F12 & $98.90 \pm 0.53$ & $6.68 \pm 0.63$ & $6.27 \pm 0.25$ & $4970 \pm 504.19$ & $71416.67 \pm 575.18$ \\
\hline F13 & $97.96 \pm 2.38$ & $7.08 \pm 0.13$ & $9.4 \pm 0.4$ & $639.333 \pm 45$ & $553 \pm 16.58$ \\
\hline F14 & $97.25 \pm 1.05$ & $6.87 \pm 0.24$ & $8.67 \pm 0.15$ & $2080.667 \pm 188$ & $1860 \pm 48.5$ \\
\hline F15 & $97.98 \pm 0.23$ & $6.82 \pm 0.26$ & $7.5 \pm 0.1$ & $3826.67 \pm 291.43$ & $13733.33 \pm 778.58$ \\
\hline F16 & $92.65 \pm 3.3$ & $6.77 \pm 0.15$ & $6.77 \pm 0.25$ & $4676.467 \pm 391.55$ & $52633.33 \pm 903.51$ \\
\hline F17 & $98.28 \pm 1.32$ & $7.05 \pm 0.13$ & $9.3 \pm 0.56$ & $707.33 \pm 35.23$ & $620 \pm 28.17$ \\
\hline F18 & $97.7 \pm 1.65$ & $6.87 \pm 0.11$ & $8.37 \pm 0.12$ & $2295.33 \pm 246.63$ & $3180 \pm 30.18$ \\
\hline F19 & $94.31 \pm 4.22$ & $6.82 \pm 0.29$ & $7.67 \pm 0.15$ & $3766 \pm 142.08$ & $10490 \pm 27.19$ \\
\hline F20 & $98.8 \pm 1.45$ & $6.59 \pm 0.19$ & $7 \pm 0.1$ & $4540.67 \pm 210.62$ & $60166.67 \pm 55.4$ \\
\hline F21 & $95.5 \pm 3.33$ & $7.13 \pm 0.1$ & $7.97 \pm 0.25$ & $1156 \pm 148.16$ & $1260 \pm 130$ \\
\hline F22 & $97.5 \pm 2.22$ & $6.93 \pm 0.15$ & $6.46 \pm 0.32$ & $4134.67 \pm 70.47$ & $9800 \pm 80$ \\
\hline F23 & $98.8 \pm 1.79$ & $6.75 \pm 0.2$ & $4.25 \pm 0.15$ & $4862.67 \pm 217.49$ & $63450 \pm 110$ \\
\hline F24 & $98.5 \pm 1.18$ & $6.8 \pm 0.2$ & $3.03 \pm 0.15$ & $5926.67 \pm 404.15$ & $309100 \pm 250$ \\
\hline
\end{tabular}

$\mathrm{S}=$ spreadability, $\mathrm{MF}=$ mucoadhesion force, $\mathrm{Cp}=$ centipoises, The results expressed as mean $\pm \mathrm{SD}(\mathrm{n}=3)$. 
The differences in the consistency of the studied systems were most likely due to formulation variables, namely the concentration of oil, surfactant/co-surfactant mixture $\left(\mathrm{S}_{\text {mix }}\right)$ and water, which modifies the gelling potential of the polymer. Thus, high concentrations of oil and $S_{\text {mix }}$ and consequently low water content, loosen the gel matrix nature of MBG formulations, while the increase of water content and absence of oil and $S_{\text {mix }}$ improved the gelling ability of polymer, particularly in the case of the conventional formulation [25].

\section{PH measurement}

All formulations were found to have satisfactory $\mathrm{pH}$. So, no irritation to nasal mucosa is expected. As in table $2, \mathrm{pH}$ of all the formulations was found to be in the range of 4.5-6.5 which is considered as nasal physiological $\mathrm{pH}$ [30]. Lysozyme is formed in the nasal secretions, which is responsible for destroying certain microbes at acidic $\mathrm{pH}$. Under alkaline $\mathrm{pH}$, lysozyme is inactive and nasal tissue is susceptible to microbial infection. It is advisable to keep the $\mathrm{pH}$ of the formulation in the range of 4.5 to $6.5[31,32]$.

\section{Drug content measurement}

Table 2 showed that sulpiride content in microemulgel and conventional hydrogel were ranged from $92.65 \pm 3.3$ to $100.14 \pm 0.33$ which complies with the pharmacopeia specifications for drug content. The data indicated the uniform distribution of sulpiride within the hydrogels and drug loss was a minimum while formulating gel $[13,16]$.

\section{Viscosity study}

For nasal gels, the viscosity is an important rheological parameter involved in its utilization and in vivo performance. Optimum viscosity value of MBGs ensures easy handling, packing, and hasslefree nasal administration. The impact of the viscosity on intranasally administered drug formulations is critical: although, a high viscosity is required to overcome MCC, yet it can impede drug spreading in the nasal cavity and its penetration through mucus. Also, too high viscosity will lead to difficult installation. On the contrary, if the viscosity is too low it will give rise to increased drainage. An optimum viscosity should always be compromised [33].

The gel viscosity depends on polymer concentration and the effect of MEs (table 2). The viscosity of the gel went on decreasing with the addition of sulpiride MEs [5]. Thus, the viscosity of the conventional hydrogel was higher than MBGs. High concentrations of oil and $S_{\text {mix }}$ and consequently low water content loosened the gel matrix nature of MBGs formulations, while the increase in water content improved the gelling ability of the polymer. Furthermore, this assumption was confirmed by the fact that sulpiride hydrogel, without oil and $S_{\text {mix }}$ in composition, presented the highest consistency, indicating that the gelling ability of the polymer was not affected [16].

The influence of the different concentrations of carbopol 940 on the viscosity of MEs was evaluated. The incorporation of carbopol 940 into MEs resulted in the significant increase of the viscosity of MEs as in table 2. The enhanced viscosity of microemulgel as compared to MEs might be attributed to the gel formulation properties of carbopol 940 as thickening agent [13]. The viscosity of the MBG is increased with increasing concentrations of carbopol 940. However, $2 \%$ carbopol 940 resulted in excessive viscosity MBG while MBG containing $1 \%$ carbopol 940 has a suitable viscosity for nasal application [29].

From all prepared MBGs, formulations prepared using ethanol as cosurfactant had the least viscosity values. Table 2 revealed that increasing in a surfactant concentration leads to an increase in the gel viscosity [14]. So, formulations without co-surfactant had a higher viscosity than other formulations. Cremophor RH-40 and labrasol, used as surfactants here, were more soluble in the external aqueous phase. Increasing the concentration of water soluble surfactant in the system increased the self-association of these amphiphilic molecules and formed different sizes and shapes of micellar aggregates. As the concentration in external phase increased, the network will be formed between the surfactant molecules, micelles, and oil droplets and the denser the network, the closer the distance between the dispersed phase and the higher the viscosity [34].

\section{Spreadability}

Application of the formulation to the affected site is more comfortable if the base spreads easily, exhibiting maximum slip and drag. The spreading of carbopol gel was found to be more uniform, and the gel spread in a circular pattern equally on all sides upon application of $500 \mathrm{~g}$ weight [28].

Table 2 revealed that spreadability is a function of viscosity and concentration of polymer, as the viscosity of the hydrogels raised the spreadability decreased and vice versa [14]. Accordingly, as carbopol 940 concentrations have increased the spreadability of the hydrogels decreased [5]. Table 2 revealed that the spreadability of MBGs was better than that of conventional hydrogels indicated by spreadability diameter of all MBGs was larger than that of conventional hydrogels. This could be due to the presence of ME globules rather than the conventional gel matrix which loose gel matrix nature of MBGs [28].

\section{Determination of mucoadhesive force (MF)}

MF was determined in terms of detachment stress by measuring the force required to detach the formulation from the mucosal surface. Carbopol 940 has a very high percentage (58\%-68\%) of carboxylic groups that gradually undergo hydrogen bonding with sugar residues in oligosaccharide chains in the mucous membrane, resulting in a formation of a strengthened network between the polymer and mucous membrane. Thus, carbopol 940 having a high density of available hydrogen bonding groups would be able to interact more strongly with mucin glycoproteins [32].

The results of MF have presented in table 2indicating that the variation in carbopol 940 concentration changes MF. MF increases with increasing concentration of mucoadhesive polymer. This is because as the concentration is increased, the more compact lattice structure is produced as well as density is increased. For that reason, more of mucoadhesive polymer remains within a fixed volume of gel to produce more hydrogen bonding between the gel and the mucin [31].

It was interesting to note that if carbopol 940 was added directly to ME without previous swelling, a loose dispersion lacked its characteristic mucoadhesion was formed. Thus, polymer swelling might affect its mucoadhesion. In addition, swollen polymers have the maximum distance between their chains leading to increased chain flexibility and efficient penetration of the substrate. If too much moisture is present and the degree of swelling is too great, a slippy mucilage results and this can be easily removed from the substrate. Maximum adhesion in vitro occurs with optimum water content during the dynamic process of adhesion [35].

Table 2 showed that conventional gel had higher mucoadhesive force than MBG. This might be due to the addition of ME decrease the viscosity of the gel. A similar result was obtained by Jitendra et al., [36] who stated that the addition of PEG 6000 to the formulations decreases the MF in a concentration-dependent manner; this might be related to the decrease in viscosity caused by PEG 6000. Also, the swelling rate of carbopol may be affected by the presence of oil, surfactant, and cosurfactant.

Moreover, water was limited in MBG while conventional hydrogels had much more water for optimum swelling of carbopol940 [35].

\section{Nasal ciliotoxicity study}

Optical microscopic results showed no mucociliary damage for MBGs as shown in fig. 2 which revealed non-ciliotoxicity profile of developed MBGs while complete cilia destruction was observed in the isopropyl alcohol treated mucosa [37]. Hence, MBGs were suitable for a nasal application. This may be due to the fact that all formulation compositions were of generally recognized as safe (GRAS) category [15]. 

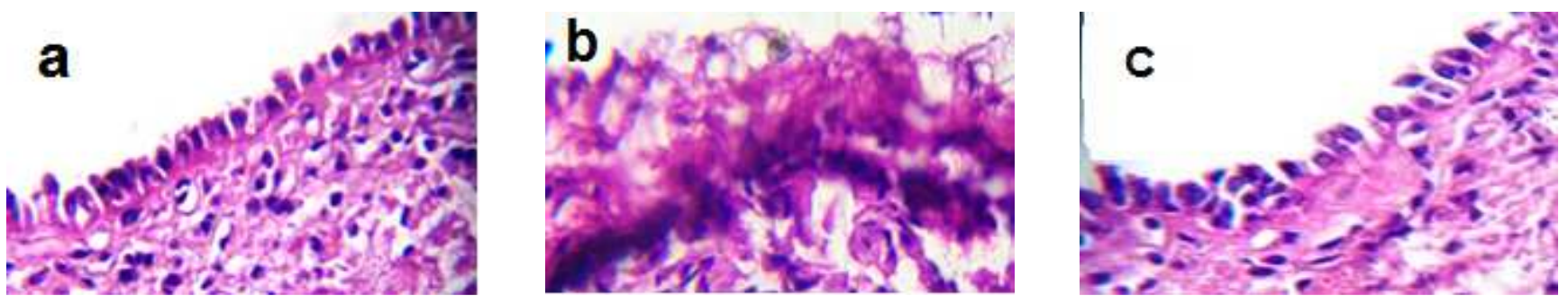

Fig. 2: Light photomicrograph of histopathological sections of sheep nasal mucosa treated with (a) Phosphate buffer saline (pH 6.8) PBS (-ve control), (b) Isopropyl alcohol (IPA)"+ve control" and (c) F14"Optimized formula."

\section{In vitro drug release studies of conventional hydrogels and MBGs}

Sink conditions were maintained for release studies $\left(\mathrm{C}_{1}<\mathrm{C}_{\mathrm{s}} \times 0.2\right)$ [35]. $\mathrm{C}_{1}$ : The final concentration of sulpiride in the medium after the complete release of the drug $(0.1 \mathrm{mg} / \mathrm{ml})$. $\mathrm{C}_{\mathrm{s}}$ : Saturation solubility of sulpiride in the PBS pH $6.8(5.65 \pm 0.49 \mathrm{mg} / \mathrm{ml})$.

This difference in release could be attributed to the different viscosity of the formulations [8]. In general, a formulation that possesses lower viscosity, expected to exhibit faster release of active ingredients [30]. It was observed that the release of sulpiride from MBGs (fig. 3) and conventional hydrogels (fig. 4) was inversely proportional to polymer concentration.

The release of drug in a semisolid dosage form depends on the viscosity of the formulation. It is also possible that the drug was trapped by higher polymer concentrations so that resistance increases to diffuse drug molecules. Additionally, drug movement area limited by the density of chain structures increased at higher polymer concentrations [5].

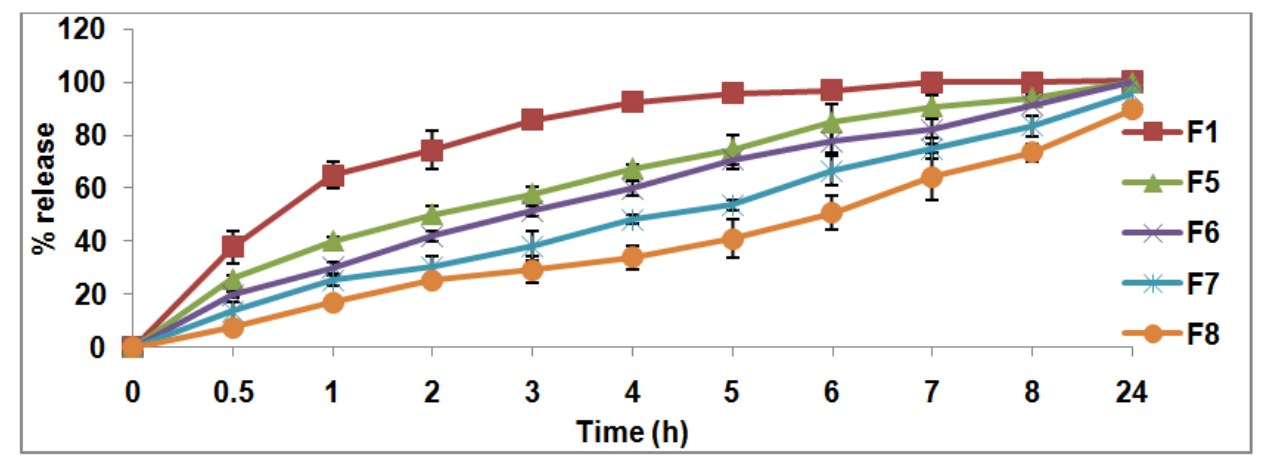

A



B



C 


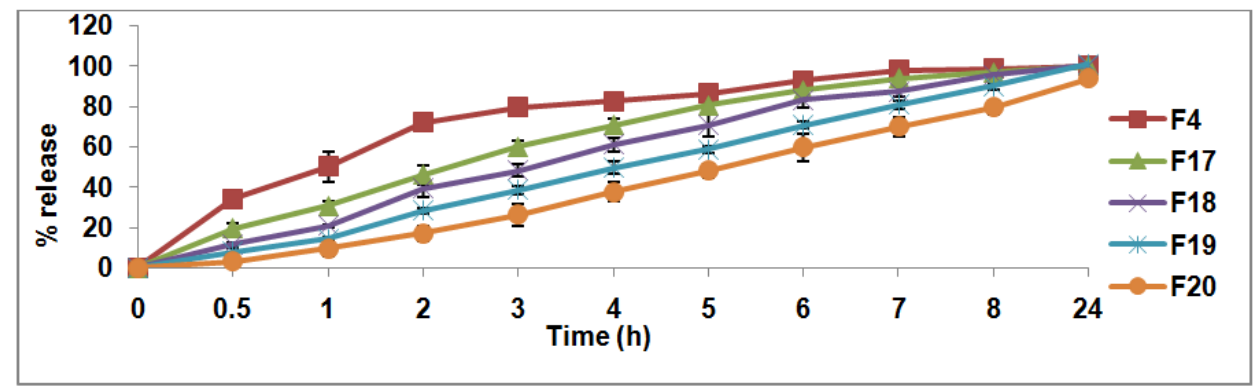

D

Fig. 3: In vitro release of sulpiride from MEs and MBGs containing different concentration of carbopol $940 *$ Each point is the mean \pm SD $(n=3)$, a: ME (F1) compared to microemulgel with different carbopol 940 concentration, b: ME (F2) compared to microemulgel with different carbopol 940 concentration, c: ME (F3) compared to microemulgel with different carbopol 940 concentration, d: ME (F4) compared to microemulgel with different carbopol 940 concentration

Fig. 3 depicted that the release of the drug from MBGs was slightly slower than its release from conventional formulation irrespective lower viscosity of MBGs. This might be due to the partitioning of the drug into the oil phase of ME gel, which retarded the drug release [38]. In the present study, MBGs were also compared with MEs in fig. 3. It was observed that the release of sulpiride from MBGs was lower than MEs, which may be due to the higher viscosity of the MBGs which may act as a barrier for drug release [19]. Due to low viscosity, MEs possessed good mobility, contributed to its freedom movements through the membrane. Also, an initial burst of the drug observed in MEs decreased after addition of carbopol that may be attributed to the difference in the viscosity of the polymers.

Hereby, from fig. 4, a reverse relationship was observed between the viscosity of different gels and the cumulative amount of drug released. Because of the loose structure of the gels prepared with low molecular weight polymers, higher amounts of the drug were released, but it is still lower than release from free sulpiride solution (SS).

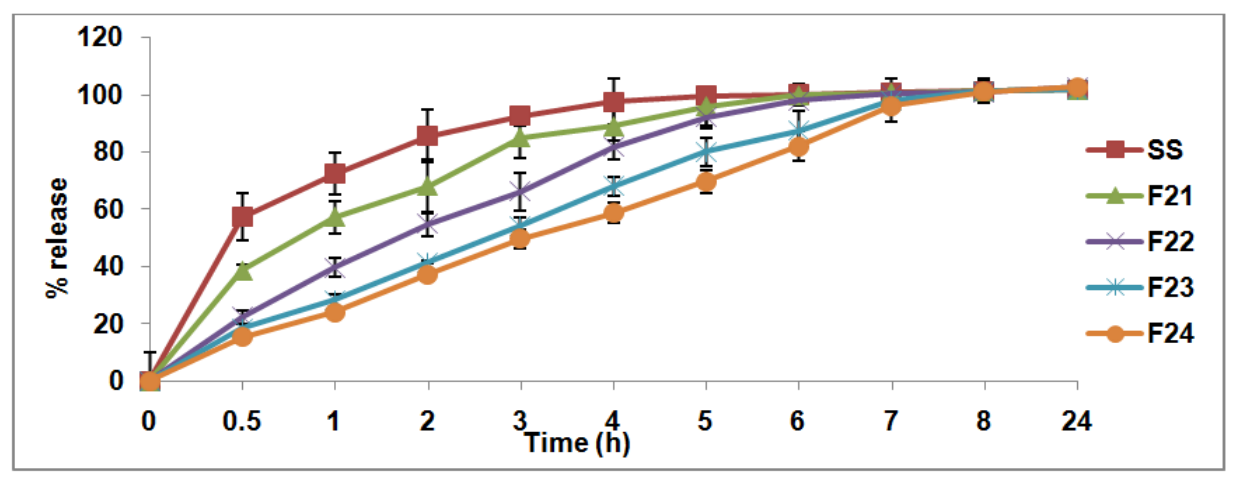

Fig. 4: In vitro release of sulpiride from conventional gel containing different concentration of carbopol $940 *$ Each point is the mean \pm SD ( $=3$ )

Table 3: Stability studies of optimized formula of sulpiride loaded MMBG (F 14)

\begin{tabular}{|c|c|c|c|c|}
\hline Months & parameter & Refrigeration & Ambient & $50^{\circ} \mathrm{C}$ \\
\hline \multirow[t]{3}{*}{0} & Drug content (\%) & $97.25 \pm 1.05$ & $97.25 \pm 1.05$ & $97.25 \pm 1.05$ \\
\hline & $\mathrm{pH}$ & $6.87 \pm 0.24$ & $6.87 \pm 0.24$ & $6.87 \pm 0.24$ \\
\hline & Viscosity & $1860 \pm 48.5$ & $1860 \pm 48.5$ & $1860 \pm 48.5$ \\
\hline \multirow[t]{3}{*}{1} & Drug content (\%) & $97 \pm 1.1$ & $96.8 \pm 2.1$ & $97.2 \pm 0.5$ \\
\hline & $\mathrm{pH}$ & $6.77 \pm 0.11$ & $6.8 \pm 0.08$ & $6.65 \pm 0.33$ \\
\hline & Viscosity & $1830 \pm 27.4$ & $1883 \pm 15.5$ & $1891 \pm 18.5$ \\
\hline \multirow[t]{3}{*}{3} & Drug content (\%) & $97.09 \pm 0.35$ & $96.14 \pm 1.65$ & $97 \pm 1.22$ \\
\hline & $\mathrm{pH}$ & $6.97 \pm 0.44$ & $6.57 \pm 0.16$ & $6.48 \pm 0.17$ \\
\hline & Viscosity & $1845 \pm 19$ & $1896 \pm 16.4$ & $1907 \pm 38.5$ \\
\hline \multirow[t]{3}{*}{6} & Drug content (\%) & $95.84 \pm 1.41$ & $96.47 \pm 1.13$ & $95.36 \pm 2.25$ \\
\hline & $\mathrm{pH}$ & $6.57 \pm 0.28$ & $6.62 \pm 0.32$ & $6.37 \pm 0.21$ \\
\hline & Viscosity & $1835 \pm 22$ & $1915 \pm 47$ & $1918 \pm 39$ \\
\hline
\end{tabular}

${ }^{*}$ The results expressed as mean $\pm \mathrm{SD}(\mathrm{n}=3)$.

\section{Stability studies}

MBGs were semi-solid gel exhibited transparent gel without precipitation of the drug, phase separation, color change or flocculation by visual inspection when they were subjected to stability at $5{ }^{\circ} \mathrm{C}, 25^{\circ} \mathrm{C}$ and $50^{\circ} \mathrm{C}$ for 6 mo [21].
Ethanol based formulae were unstable during storage. Color change in some formulae into ivory white and start to dry and become harder due to slow evaporation of ethanol at a higher temperature. Also, phase separation was observed in these formulations. As shown in table 3 , there are negligible changes in the parameters 
such as drug content, $\mathrm{pH}$ value, and viscosity. These parameters did not change significantly after MBGs were stored for 6 mo which implied MBGs were stable for storage. No degradation of sulpiride was observed during 6 mo. occurred under centrifugation at 13000 rpm for $15 \mathrm{~min}$.

The centrifuge tests showed that MBGs had good physical stability with no phase separation and still maintain a good thermodynamic stability similar to MEs [20]. It is possible that the gel network increased the viscosity of MEs but have no influence on the $\mathrm{o} / \mathrm{w}$ structure of MEs. Moreover, the addition of a mucoadhesive polymer may further stabilize the system since it increased negative charge of the system [11].

\section{Ex-vivo permeation}

As expected, the drug permeation rate through the excised nasal mucosa was clearly slower than that through the artificial membrane, due to the major barrier effect exerted by the biological membrane [24] as depicted in fig. 5. It was surprising that PEG 400 and/or ethanol in sulpiride solution (SS) could enhance drug permeation through the nasal mucosa. PEG400 as a permeation enhancer increases the rate as well as the extent of the release, which may be due to its solubilizing effect as well as its penetration enhancing the effect. It was reported the permeation is further increased with the use of PEG than tween 80 [39].

To evaluate the superiority of MBGs, a conventional gel containing a plain sulpiride (without MEs) was also studied and the obtained results indicate that permeation of sulpiride from MBGs is better than that from conventional gel containing plain sulpiride, which could be assigned to the concept that, these hydrogels were ME based and of less viscous nature [5]. This agrees with the result obtained by Naeem et al., [21] who stated that the flux of ME and MBG was greater than that of polymer based gels containing a hydro-alcoholic solution. Also, drug permeation from the conventional mucoadhesive gel was higher than in SS. This observation can be explained by the fact that MBG formulations contain a higher surfactant concentration which increases the solubility of the drug, also contain labrasol, cremophor and transcutol HP that act as permeation enhancers $[5,10]$. Initially, the result showed that addition of a gelling agent into ME decreased the permeability of sulpiride. However, (F14) MBG showed higher drug diffusion as compared to ME after $24 \mathrm{~h}$. The drug release from hydrogel explained as diffusion at an initial stage and then a combination of diffusion and degradation at a later stage. This may be explained by bio-adhesion and absorption enhancement property of carbopol 940 across the mucosal membrane [19]. Carbopol has a penetration enhancing the effect by opening the tight junctions of the nasal mucosa. Thereby, carbopol 940 might facilitate transport of drugs via a paracellular pathway [37]. Also, carbopol tends to adhere to the mucosa and form hydrogen bonding with sugar residues in oligosaccharide chains in the mucous membrane thereby causing increased contact and hence increased diffusion [40]. Similarly, permeation of ropinirole was higher from the formulation containing $1 \%$ chitosan $\mathrm{HCl}$ than plain nasal solution due to the contribution of paracellular transport through tight junctions. However, a further increase in concentration to $2 \%$ did not improve the drug permeation [17]. The moderate viscosity of the formulation can improve drug absorption across the nasal mucosa, while higher viscosity can decrease the drug penetration rate across the mucus layer, and lead to the irritation of the nasal mucosa. Higher viscosity is preferred as it increases residence time,but permeation rate decreases with too high viscosity and hence the formulation should have moderate viscosity [33].

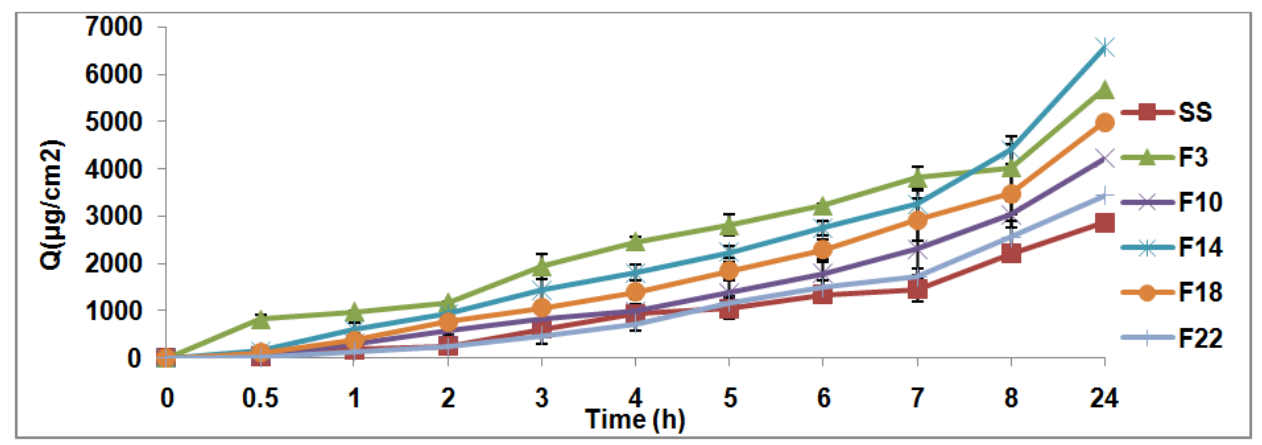

Fig. 5: Ex-Vivo permeation of sulpiride from different MBGs compared to SS and ME (F3) Q: cumulative amount of drug permeated per unit surface area $\left(\mu \mathrm{g} / \mathrm{cm}^{2}\right) *$ Each point is the mean $\pm S D(n=3)$

The permeation parameters are listed in table 4 . The comparatively higher flux after $24 \mathrm{~h}\left(\mathrm{Q}_{24}\right)$ of MBGs and ME could be due to increased diffusion coefficient of the drug. Moreover, smaller globule size of ME provides a larger area for drug permeation and high drug concentration in the affected area results in a larger concentration gradient, which is essential for efficient drug delivery through natural membranes [40]. The highly homogeneous and extremely fine dispersion of the drug completely dissolved into the nano-sized droplets of the $\mathrm{o} / \mathrm{w} \mathrm{ME}$, probably allowed to maximize the drug concentration gradient at the level of the barrier membrane. The solubilizing and carrier effect of ME toward sulpiride is also be taken into account [24]. The lag time of sulpiride loaded MBG was found to be in the range $(1.61-1.78 \mathrm{~h})$, which was significantly $\left({ }^{* *} \mathrm{P}<0.05\right)$ higher than that of ME $(0.25 \pm 0.05 \mathrm{~h})$. There was no significant difference in the lag time between different MBG. MBGs showed a sustained permeation of sulpiride, compared to ME. This phenomenon could be elucidated with the release retarding effect of the polymeric matrix, mainly due to the increased viscosity stemming from carbopol gelation [3].

Overall, shorter lag times were observed for MEs based formulas than conventional formula and drug solution. It is assumed that by incorporation into the colloidal carrier, a better contact with the membrane is achieved, which could be one of the reasons why the effect appeared sooner, resulting in shorter lag times. Also, it was reported that microemulgel are known to provide faster drug release than conventional formulations [28].

A higher concentration of carbopol 940 (2\%) resulted in an excessive viscosity, high mucoadhesive strength, and less diameter in spreadability test. On the other hand, a lower concentration of carbopol $940(0.5 \%)$ resulted in high fluidity and very loose preparation. So, MBG formulation based on $1 \%$ carbopol 940 was selected for further testing.

\section{Pharmacodynamic study (Paw test)}

Table 5 revealed that F14 administered intranasally (IN) showed higher HRT values as compared to F3 (intranasal) and F3 (IV) indicating the superiority of intranasal MBG over the intranasal and intravenous administration of ME [22]. This suggested better brain uptake of the drug by intranasal route as MBG when compared with other sulpiride formulations. Furthermore, table 5 showed that F14 showed higher HRT values when compared with F3, both administered intranasally, indicating the superiority of intranasal 
MBG over MEs and clearly, demonstrates the role of carbopol 940 (mucoadhesive agent) that decrease MCC, thereby prolonging the residence time, and is also suggested to play a role in the enhancement of penetration of the formulation across the cells [22]. From these results, it can reasonably be concluded that brain targeting could be achieved by MBG. This finding may be attributed to direct nose-to-brain transport bypassing the BBB that may have occurred due to the unique connection between the nose and CNS [40]. It is known that mucoadhesive additives may improve drug absorption by reducing the mucociliary clearance rate and by increasing the residence time of the drug formulation in the nasal cavity. Furthermore, some mucoadhesive polymer-containing systems may directly change epithelial tight junctions and increase drug absorption and bioavailability [37].

Table 4: Permeation parameters of conventional gel and MBGs

\begin{tabular}{lllllll}
\hline Parameters & SS & F3 & F10 & F14 & F18 & F22 \\
\hline $\mathrm{Q}_{24}\left(\mu \mathrm{g} / \mathrm{cm}^{2}\right)$ & $2870.72 \pm 191.5$ & $5686.48 \pm 309.13$ & 4222.6 & 6575.4 & 5088.76 & 3436.95 \\
& & & \pm 272.5 & \pm 288.94 & \pm 439.83 & \pm 127.04 \\
$\mathrm{~J}_{\mathrm{ss}}\left(\mu \mathrm{gcm}^{-2} \mathrm{~h}^{-1}\right)$ & 291.95 & 499.65 & 417.72 & 580.33 & 511.62 & 369.01 \\
& & & \pm 35.31 & \pm 19.36 & \pm 20.00 & \pm 6.57 \\
$\mathrm{~K}_{\mathrm{P}}\left(\mathrm{cm}^{-2} \mathrm{~h}^{-1}\right)$ & 29.2 & 50 & 41.77 & 58.03 & 51.16 & 36.9 \\
$\mathrm{Lag} \mathrm{time}(\mathrm{h})$ & 1.5 & 0.25 & 1.78 & 1.61 & 1.78 & 2.01 \\
& & \pm 0.05 & \pm 0.10 & \pm 0.17 & \pm 0.20 & \pm 0.08 \\
$\mathrm{ER}$ & - & 1.71 & 1.43 & 1.99 & 1.75 & 1.27 \\
\hline
\end{tabular}

Cumulative amount after $24 \mathrm{~h}\left(\mathrm{Q}_{24}\right)$, Steady-state flux $\left(\mathrm{J}_{\mathrm{ss}}\right)$, a permeability coefficient $\left(\mathrm{K}_{\mathrm{P}}\right)$ and enhancement ratio(ER). The results expressed as mean \pm SD $(n=3)$.

Table 5: Pharmacodynamic study of sulpiride formulations in rat

\begin{tabular}{lllllll}
\hline Parameter & Control & SS (IN) & F3 (IN) & F3 (IV) & F14 (IN) & Haloperidol (IP) \\
\hline FRT(s) & $4.6 \pm 1.14$ & $4.2 \pm 1.1$ & $3.6 \pm 0.89$ & $4.8 \pm 0.84$ & $5.2 \pm 0.84$ & $18 \pm 5.15$ \\
HRT(s) & $3.6 \pm 0.89$ & $9.5 \pm 2.07$ & $13.2 \pm 2.39$ & $15.4 \pm 1.82$ & $27.2 \pm 3.27$ & $36.4 \pm 6.23$ \\
\hline
\end{tabular}

FRT: (Forelimb Retraction Time), HRT: (Hind limb Retraction Time), IN=intranasal, IV= intravenous, IP=intraperitoneal, The results are mean values (in second) \pm SD derived from three different animals $(\mathrm{n}=3)$.

\section{CONCLUSION}

Carbopol 940 provides excellent adhering, constant releasing formulation and the optimum viscosity for nasal application. Gel viscosity did not cause significant unfavorable effects on the drug permeation. Finally, the combination of mucoadhesive polymer and MEs as co-enhancer improves membrane permeability and bioavailability in the nasal delivery of drugs.

\section{CONFLICTS OF INTERESTS}

Declared none

\section{REFERENCES}

1. Qian S, Wong YC, Zuo Z. Development, characterization and application of in situ gel systems for intranasal delivery of tacrine. Int J Pharm 2014;468:272-82.

2. Dixit GR, Mathur VB. Microemulsions: a platform for improvement of solubility and dissolution of poorly soluble drugs. Asian J Pharm Clin Res 2015;8:7-17.

3. Zhao L, Wang Y, Zhai Y, Wang Z, Liu J, Zhai G. Ropivacaine loaded microemulsion and microemulsion-based gel for transdermal delivery: preparation, optimization, and evaluation. Int J Pharm 2014;477:47-56.

4. Watanabe K, Sawano T, Jinriki T, Sato J. Studies on intestinal absorption of sulpiride: intestinal absorption of sulpiride in rats. Biol Pharm Bull 2014;27:77-81.

5. Pandey S, Das U, Patil A. Formulation and ex-vivo evaluation of metronidazole microemulsion are loaded hydrogel for prevention of periodontitis. J Pharm Invest 2014;44:225-36.

6. Khattab A, Ismail S. Formulation and evaluation of oxiconazole nitrate mucoadhesive nanoemulsion-based gel for the treatment of vaginal fungal infection. Int J Pharm Pharm Sci 2016;8:33-40.

7. El-Badry M, Fetih G, Shakeel F. Comparative topical delivery of antifungal drug croconazole using liposome and micro-emulsionbased gel formulations. Drug Delivery 2014;21:34-43.

8. Çelebi N, Ermiş S, özkan S. Development of topical hydrogels of terbinafine hydrochloride and evaluation of their antifungal activity. Drug Dev Ind Pharm 2015;41:631-9.
9. Yadav SA, Singh DK, Poddar SS. Formulation, in vitro and in vivo evaluation of nanoemulsion gel for transdermal drug delivery of nimodipine. Asian J Pharm Clin Res 2015;8:119-24.

10. ÜstündağOkur N, Çağlar EŞ, Arpa MD, Karasulu HY. Preparation and evaluation of novel microemulsion-based hydrogels for dermal delivery of benzocaine. Pharm Dev Technol 2016;7:1-11.

11. Mou D, Chen H, Du D, Mao C, Wan J, Xu H, et al. Hydrogelthickened nanoemulsion system for topical delivery of lipophilic drugs. Int J Pharm 2008;353:270-6.

12. Acharya SP, Pundarikakshudu K, Panchal A, Lalwani A. Preparation and evaluation of transnasal microemulsion of carbamazepine. Asian J Pharm Sci 2013;8:64-70.

13. Dhingani A, Patel J, Garala K, Dharamsi A. Design and development of microemulsion based transdermal gel of lercanidipine hydrochloride by assimilation of rotatable central composite design and principal component analysis. Pharm Nanotechnol 2013;1:290-305.

14. Pednekar A, Dandagi P, Gadad A, mastiholimath V. Formulation and characterisation of meloxicam are loaded emulgel for topical application. Int J Pharm Pharm Sci 2015;7:216-22.

15. Mandal SD, Mandal S, Patel J. Brain targeting efficiency of curcumin loaded mucoadhesive microemulsion through intranasal route. J Pharm Invest 2016;46:179-88.

16. Coneac G, Vlaia V, Olariu I, Muţ AM, Anghel DF, Ilie C, et al. Development and evaluation of new microemulsion-based hydrogel formulations for topical delivery of fluconazole. AAPS PharmSciTech 2015;16:889-904.

17. Khan S, Patil K, Bobade N, Yeole P, Gaikwad R. Formulation of intranasal mucoadhesive temperature-mediated in situ gel containing ropinirole and evaluation of brain targeting efficiency in rats. J Drug Target 2010;18:223-34.

18. Shah BM, Misra M, Shishoo CJ, Padh H. Nose to brain microemulsion-based drug delivery system of rivastigmine: formulation and ex-vivo characterization. Drug Delivery 2014;22:918-30.

19. Sood S, Jain K, Gowthamarajan K. Optimization of curcumin nanoemulsion for intranasal delivery using the design of the experiment and its toxicity assessment. Colloids Surf B 2014;113:330-7. 
20. Sahoo S, Pani NR, Sahoo SK. The microemulsion based topical hydrogel of sertaconazole: formulation, characterization, and evaluation. Colloids Surf B 2014;120:193-9.

21. Naeem M, Ur Rahman N, Tavares GD, Barbosa SF, Chacra NB, Löbenberg $\mathrm{R}$, et al. Physicochemical, in vitro and in vivo evaluation of flurbiprofen microemulsion. Ann Acad Bras Cienc 2015;87:1823-31

22. Kumar M, Misra A, Babbar AK, Mishra AK, Mishra P, Pathak K. Intranasal nanoemulsion-based brain is targeting drug delivery system of risperidone. Int J Pharm 2008;358:285-91.

23. Ellenbroek BA, Zhang X, Jin GZ. Effect of (-) stipholidine in an animal model of schizophrenia. Acta Pharmacol Sin 2006;27:1111-8.

24. Mura P, Bragagni M, Mennini N, Cirri M, Maestrelli F. Development of liposomal and microemulsion formulations for transdermal delivery of clonazepam: effect of randomly methylated $\beta$-cyclodextrin. Int J Pharm 2014;475:306-14.

25. Olariu I, Coneac G, Vlaia L, Vlaia V, Anghel DF, Ilie C, et al. Development and evaluation of microemulsion-based hydrogel formulations for topical delivery of propranolol hydrochloride. Digest J Nanomaterials Biostructures 2014;9:395-412.

26. Pillai AB, Nair JV, Gupta NK, Gupta S. Microemulsion-loaded hydrogel formulation of butenafine hydrochloride for improved topical delivery. Arch Dermatol Res 2015;307:625-33.

27. Sharma K, Bedi N. Microemulsion based hydrogel of mycophenolate mofetil for the treatment of psoriasis. Curr Trends Biotechnol Pharm 2014;8:359-71.

28. Patel MR, Patel RB, Parikh JR, Patel BG. Novel microemulsionbased gel formulation of tazarotene for therapy of acne. Pharm Dev Technol 2015;3:1-12.

29. Luo $\mathrm{M}$, Shen $\mathrm{Q}$ Chen J. Transdermal delivery of paeonol using cubic gel and microemulsion gel. Int J Nanomed 2011;6:1603-10.

30. Priya S, koland M, kumari SN. Nanoemulsion components screening of quetiapine fumarate: effect of surfactant and cosurfactant. Asian J Pharm Clin Res 2015;8:136-40.

31. Bhandwalkar MJ, Avachat AM. Thermoreversible nasal in situ gel of venlafaxine hydrochloride: formulation, characterization, and pharmacodynamic evaluation. AAPS PharmSciTech 2013;14:101-10.
32. Pathan IB, Chudiwal V, farooqui I, Shingare P. Formulation design and evaluation of nasal in situ gel as a novel vehicle for azelastine hydrochloride. Int J Drug Delivery 2013;5:284-90.

33. Furubayashi T, Inoue D, Kamaguchi A, Higashi Y, Sakane T. Influence of formulation viscosity on drug absorption following nasal application in rats. Drug Metab Pharmacokinet 2007; 22:206-11.

34. Arora R, Aggarwal G, Harikumar SL, Kaur K. Nanoemulsion based hydrogel for enhanced transdermal delivery of ketoprofen. Adv Pharm 2014;5:1-14.

35. Bansal K, Rawat MK, Jain A, Rajput A, Chaturvedi TP, Singh S. Development of satranidazole mucoadhesive gel for the treatment of periodontitis. AAPS PharmSciTech 2009;10:716-23.

36. Jitendra VS, Kailas KM, Remeth JD, Vijay DH, Niranjan SM. In situ mucoadhesive nasal gels of metoclopramide hydrochloride: pre-formulation and formulation studies. J Pharm Res 2008;1:88-96.

37. Pathak R, Prasad Dash R, Misra M, Nivsarkar M. Role of mucoadhesive polymers in enhancing delivery of nimodipine microemulsion to the brain via the intranasal route. Acta Pharm Sin B 2014;4:151-60.

38. Derle DV, Sagar BSH, Pimpale R. Microemulsion as a vehicle for transdermal permeation of nimesulide. Indian J Pharm Sci 2006;68:622-5.

39. Sahu BP, Sharma HK, Das MK. Development and evaluation of a mucoadhesive nasal gel of felodipine prepared with the mucoadhesive substance of Dilleniaindica L. Asian J Pharm Sci 2011;5:175-87.

40. Patel RB, Patel MR, Bhatt KK, Patel BG. Paliperidone-loaded mucoadhesive microemulsion in the treatment of schizophrenia: formulation consideration. J Pharm Innov 2013;8:195-204.

\section{How to cite this article}

- $\quad$ Abdallah Mohamed Ayoub, Mahmoud Mokhtar Ibrahim, Marwa Helmy Abdallah, Mahmoud A Mahdy. Intranasal microemulgel as a surrogate carrier to enhance low oral bioavailability of sulpiride. Int J Pharm Pharm Sci 2016;8(10):188-197. 